\title{
EFFECT OF POROUS SILICA AS A DRUG CARRIER ON THE RELEASE RATE OF NAPROXEN FROM EMULGEL
}

Ahmed Ismail, K. I. Saleh*, M.A. Ibrahim and S. Khalaf

Department of Pharmaceutics, Faculty of Pharmacy, Al-Azhar University, Assiut, Egypt

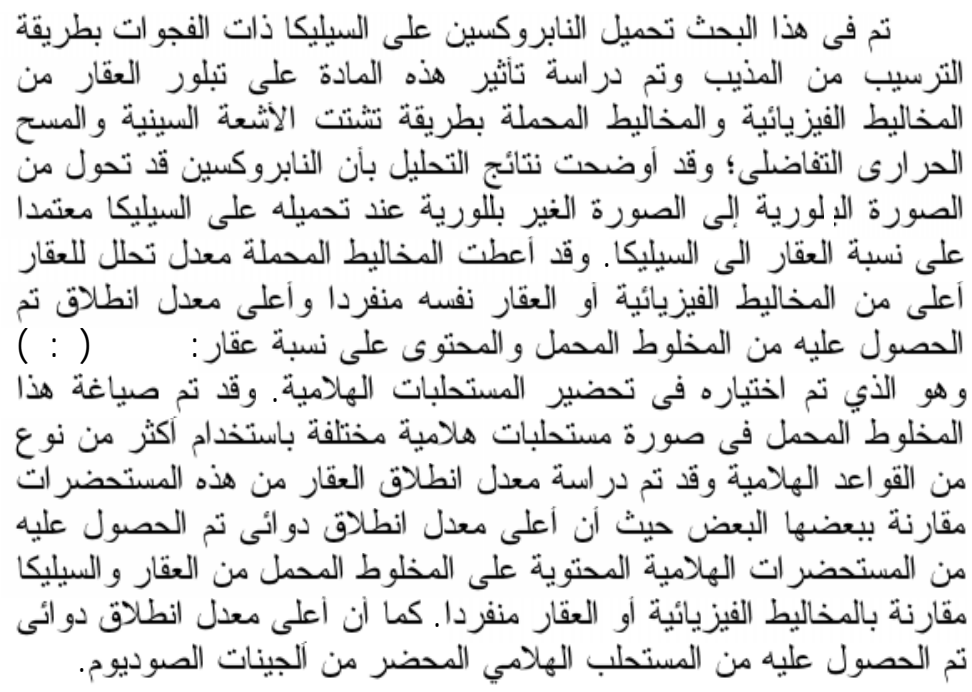

Naproxen was loaded on Florite $R$ (porous silica) by solvent deposition technique. The physical and loaded drug mixtures were examined by $x$-ray diffractometry and differential scanning calorimetry (DSC). The in-vitro dissolution of the drug from loaded and physical mixture was studied. The best formula which contains (1:2) drug:adsorbent ratio was formulated into emulgel using different gelling agents. The in-vitro release of naproxen from the selected emulgel bases was carried out. X-ray and DSC results revealed that the drug was changed from the crystalline to amorphous state depending on drug to carrier ratio since the complete transformation was obtained from the loaded mixture containing (1:2) drug:Florite $R$ ratio. Transformation of the drug to the amorphous state in the loaded mixture led to an enhancement of both drug dissolution and in-vitro release rates.

Received in 6/3/2006 \& Accepted in 5/7/2006

* Corresponding author 


\section{INTRODUCTION}

Naproxen is a candidate of non steroidal anti-inflammatory drug which is used in the treatment of arthritis and various inflammatory disorders. ${ }^{1}$ It is practically water insoluble, ${ }^{2}$ resulting in poor absorption. The dissolution rate of the drug is influenced by its physical state, including polymorphism, hydrate or solvate formation and also the degree of crystallinity. ${ }^{3}$

Numerous attempts ${ }^{4-6}$ have been made to improve the dissolution rate of poorly water-soluble drugs. Surface adsorption is one of the methods used to reduce the drug particle size by increasing the surface area available to the dissolution medium. ${ }^{7}$ The technique of surface adsorption was first reported by Mankhouse and Lach. Recently, this technique has been employed to improve the dissolution characteristics of certain drugs. ${ }^{8 \& 9}$

Porous silica gel or porous calcium silicate, which called Florite $\mathrm{R}$ is hydrophilic adsorbent of globular structure. ${ }^{10}$ Porous calcium silicate is widely used as a drug carrier in various pharmaceutical preparations for its excellent flowability, mouldability, drug stabilization and release characters. ${ }^{11 \& 12}$ The use of colloidal and porous silica has recently attracted considerable attention in the pharmaceutical field of stability, enhancement of dissolution and bioavailability. ${ }^{13}$

The aim of the present study was to evaluate the effect of porous silica as a drug carrier on the physico- chemical properties and the dissolution rate of naproxen. Another goal of this work is to prepare naproxen emulgel and evaluate the effect of porous silica on the in vitro release rate of the drug from the emulgel bases.

\section{EXPERIMENTAL}

\section{Materials}

Naproxen (kindly provided by Misr Pharmaceutical Co., Egypt); Porous calcium silicate (Tokuyama Soda, Tokyo, Japan) was used after drying in vacuum at $120^{\circ}$ for $3 \mathrm{hr}$; Standard cellophane membrane of molecular cut range $\approx 12000$ ) (Sigma Chem. Co., USA); Hydroxypropylmethylcellulose (HPMC), (Aldrich Chem. Co., Inc., USA); Methylcellulose (MC), (BDH Chemical LTD, Poole Englad); Carboxymethylcellulose, Sodium alginate and Castor oil (El-Nasr Pharm. Chem. Co., Cairo, Egypt). All other materials were of analytical grade.

\section{Equipment}

Double beam spectrophotometer, UV-1601 (Shimadzu Co., Japan); Vibrational sieve shaker, RX-86-1 (Cole Pulmer Instrument Co., USA); X-ray diffractometer (Philips Co., 1710 Netherland); Differential scanning calorimeter, DSC-50, equipprd with a computerized data station (Shimadzu Co., Japan); Dissolution test apparatus, SR II, 6 flask (Hanson Research Co., USA); Vacuum oven drier, STP-200 (Zeamil Horyzont Co., Poland); Thermostatically controlled shaking water 
bath (Kotterman Labortechnik GmbH, Germany); Homogenizer (Mechanika Precyzyna- MPN-09, Poland); pH meter Ama digital (Ama Co., Germany).

Methods

Preparation of naproxen physical mixtures

Naproxen, which had a particle size of 125-200 $\mathrm{m}$ and dried porous silica (Florite $\mathrm{R}$ ) were mixed in various ratios (1:0.5, 1:1 and 1:2 drug to Florite $\mathrm{R}$ ratios) using mortar and pestle in dark and dry conditions to avoid oxidation of the drug. Mixing was done gently for five minutes avoiding any grinding effect on the mixture.

\section{Preparation of naproxen loaded adsorbates}

The loaded mixtures of naproxen using different carriers were prepared by solvent deposition method. The calculated amount of Florite $\mathrm{R}$ was added to the methanolic solution of naproxen to give the desired drug: adsorbent ratios. Adsorbate systems containing $1: 0.5,1: 1$ and $1: 2 \mathrm{w} / \mathrm{w}$ drug: adsorbent ratios were prepared. Methanol was evaporated at room temperature and its complete removal was carried out over anhydrous calcium chloride up to constant weight. The samples were then scrapped and the particle size of each prepared mixture was reduced to the initial size of the used adsorbents and then assayed for their drug content. Only those samples containing $100 \pm$ $5 \%$ of naproxen were used for further studies.
Physicochemical characterization of the prepared loaded and physical mixtures

Powder X-ray diffraction studies

The $\mathrm{x}$-ray diffraction patterns for different samples with particle size range (125-200) $\mu \mathrm{m}$ were determined using the Philips 1710 automated diffractometer. The relation was provided by $\mathrm{CuK} \alpha$ radiation operating at $40 \mathrm{KV}$ and current of 30 $\mathrm{mA}(\lambda \mathrm{K} \alpha=1.5418 \AA)$. The system was calibrated using standard polycrystalline silicon. The differential patterns were achieved using continuous scan mode with $2 \theta$ ranging from $4^{\circ}$ to $60^{\circ}$. The output data achieved were represented by $2 \theta$, $\mathrm{d} \AA$, intensities were determined via the microprocessor of the PW / 1710.

\section{Differential scanning calorimetry (DSC)}

The DSC thermograms were recorded on a Schimadzu DSC-50 calorimeter with samples weight (about $5 \mathrm{mg}$ ). The procedure involves heating the sample contained in an aluminum pan and a similar empty reference pan at a predetermined temperature range. The samples were scanned at scanning rate of $10 \% \mathrm{~min}$ up to $200^{\circ}$ under dry nitrogen stream at flow rate of $40 \mathrm{ml} / \mathrm{min}$.

\section{In-vitro dissolution studies}

The dissolution rate of naproxen from its loaded and physical mixtures as well as the untreated drug was studied using USP dissolution apparatus. Accurately weighed amount equivalent to $40 \mathrm{mg}$ of the 
drug was dispersed on the medium $(500 \mathrm{ml}$ citrate buffer, pH 5.03 at 37 $\pm 0.2^{\circ}$ ), which was immediately stirred at $60 \mathrm{rpm}$. At appropriate time intervals (after 5, 10, 15, 30, 45, 60, 75 and $90 \mathrm{~min}$.) $5 \mathrm{ml}$ sample was withdrawn by a pipette fitted with a cotton plug at its terminal end. The sample was diluted to a suitable volume with a buffer solution and the absorbance was measured spectrophotometrically at $271 \mathrm{~nm}$. Equal volume of fresh dissolution medium, prewarmed at $37^{\circ}$, was added to the dissolution medium so as to keep the volume of the dissolution medium constant. The cumulative amount of the drug dissolved during the $\mathrm{n}^{\text {th }}$ sample was calculated.

\section{Formulation of naproxen adsorbate as emulgel \\ Preparation of emulgels}

Emulgels used in the present study were $\mathrm{o} / \mathrm{w}$ emulsions, composed of vegetable oil emulsified in aqueous medium with the aid of synthetic emulsifier such as tween ${ }^{\circledR}$ and then mixed with a gelling agent to obtain creamy consistency.

One gram of naproxen or its equivalent from adsorbate containing (1:2) drug to Florite $\mathrm{R}$ ratio was dissolved in the oil phase and mixed thoroughly with water $(10 \mathrm{ml})$ and tween $60^{\circledR}$ as emulsifying agent. A gelling agent was allowed to swell in the remaining water. A drug-loaded emulsion was gelled with a preswollen gel and then was treated with a homogenizer for 5 minutes till a homogenous emulgel is obtained. ${ }^{14}$
Table 1, illustrates the composition of the tested emulgel bases.

Table 1: Composition of emulgel bases.

\begin{tabular}{||l|c|c|c|c||}
\hline \multirow{2}{*}{ Component } & \multicolumn{4}{|c|}{$\begin{array}{c}\text { Concentration } \\
\text { (\% w/w) }\end{array}$} \\
\cline { 2 - 5 } & I & II & III & IV \\
\hline Castor oil & 20 & 20 & 20 & 20 \\
Tween $60^{\circledR}$ & 2 & 2 & 2 & 2 \\
Na-CMC & 2 & --- & --- & --- \\
HPMC & --- & 2.5 & --- & --- \\
MC & --- & --- & 5 & --- \\
Na-alginate & --- & --- & --- & 5 \\
Dist. water & 76 & 75.5 & 73 & 73 \\
\hline \hline
\end{tabular}

\section{In-vitro release of naproxen from the prepared emulgel formulations}

From the previous studies in the preformulation part, the results revealed that the base containing 1:2 w/w drug to Florite R ratio showed the highest dissolution rate. On the other hand, DSC and x-ray studies confirmed the transformation of naproxen from crystalline to amorphous state in this ratio. Hence, this base was selected in order to study the release of naproxen from the emulgel formulations.

One gram from each emulgel formulation containing $10 \mathrm{mg}$ pure drug was placed on a semi permeable cellulosic cellophane membrane previously soaked in distilled water for $24 \mathrm{hrs}$. The loaded membrane with the sample of emulgel was stretched over one end of an open glass tube $\left(6.63 \mathrm{~cm}^{2}\right.$ area).

The membrane was clipped tightly over the tube end by means of a 
cotton thread. The tube was suspended so that the membrane was $1 \mathrm{~cm}$ just below the surface of $100 \mathrm{ml}$ citrate buffer of $\mathrm{pH} 5.03$ at $34 \pm 0.3^{\circ}$ contained in $250 \mathrm{ml}$ beaker and shaked in a thermostatically controlled water bath kept at $60 \mathrm{rpm}$. At specified time intervals, $2 \mathrm{ml}$ samples were withdrawn and replaced with an equal volume of a fresh release medium. The amount of naproxen released was assayed at $271 \mathrm{~nm}$.

\section{RESULTS AND DISCUSSION}

Powder x-ray diffractometry was utilized to study the crystallographic nature of adsorbate mixture, physical mixture and plain drug. ${ }^{15}$ The major $x$-ray diffraction peaks of naproxen were observed at $2 \theta$ at $5.59^{\circ}$, $18.9^{\circ}, 22.2^{\circ}, 22.5^{\circ}$ and $28^{\circ} \AA$ with relative intensities of $100,65,48,92$, 59, and 53, respectively (Fig. 1).

The x-ray diffraction pattern of the physical mixtures of naproxen and Florite $\mathrm{R}$ in both (1:1) and (1:2) ratios showed the diffraction peak of low intensity. Similar finding was obtained in case of $(1: 1)$ loaded mixture (Fig. 1).

Regarding the adsorbate containing naproxen/FloriteR (1:2) ratio, which illustrated in Fig.2, x-ray diffraction peaks of the drug have completely disappeared. It can be assumed from these results that the loading of naproxen on porous silica resulted in its transformation from a crystalline to an amorphous state (mixture containing 1:2 w/w ratio) or just reduction of its crystallinity (mixture containing 1:1 w/w ratio).

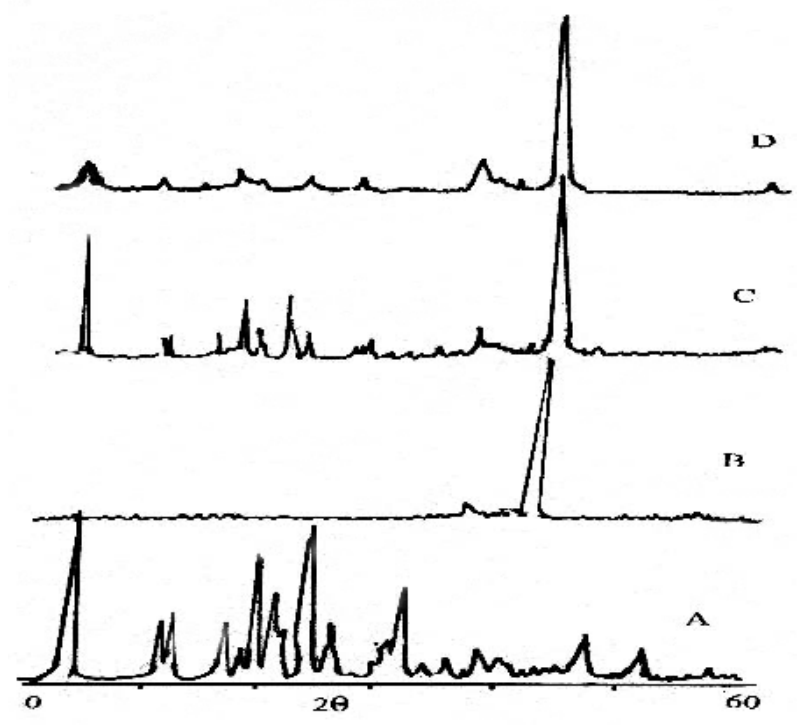

Fig. 1: X-ray diffraction patterns of (1:1) naproxen /Florite R Systems, where:
A- Plain drug
B- Florite $\mathrm{R}$
C- Physical mixture
and
D- Adsorbate 


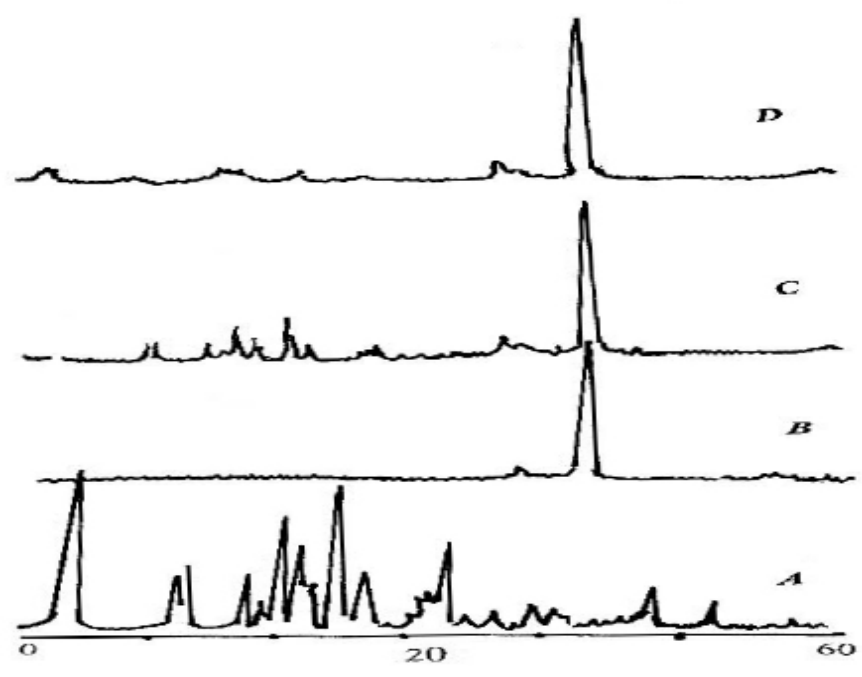

Fig. 2: X-ray diffraction patterns of (1:2) naproxen /Florite R systems, where:
A- Plain drug
B- Florite R
C- Physical mixture
and
D- Adsorbate

Differential scanning calorimetry has been used for characterizing the state of the drug and studying its interaction with carriers. ${ }^{15 \& 16}$ Fig. 3, illustrated DSC thermograms of naproxen and its physical mixtures as well as the adsorbates with Florite $\mathrm{R}$ in both $1: 1$ and 1:2 w/w drug: adsorbent ratios. Naproxen showed an endothermic peak at $156^{\circ}$ due to melting of drug crystals (Fig. 3, trace A), but the DSC curve of Florite R is characterized by absence of peaks within the temperature range employed (Fig. 3, trace B). The thermograms of physical mixtures and (1:1) loaded mixture showed the same drug characteristic peak but with low intensity and a little shift to lower degree of temperature.

In case of the loaded mixture of 1:2 w/w drug: Florite $\mathrm{R}$ ratio, the DSC curve showed no endothermic peak at the melting region of drug crystals and this confirms the presence of the drug in the amorphous state at this ratio (Fig. 3, trace F) 


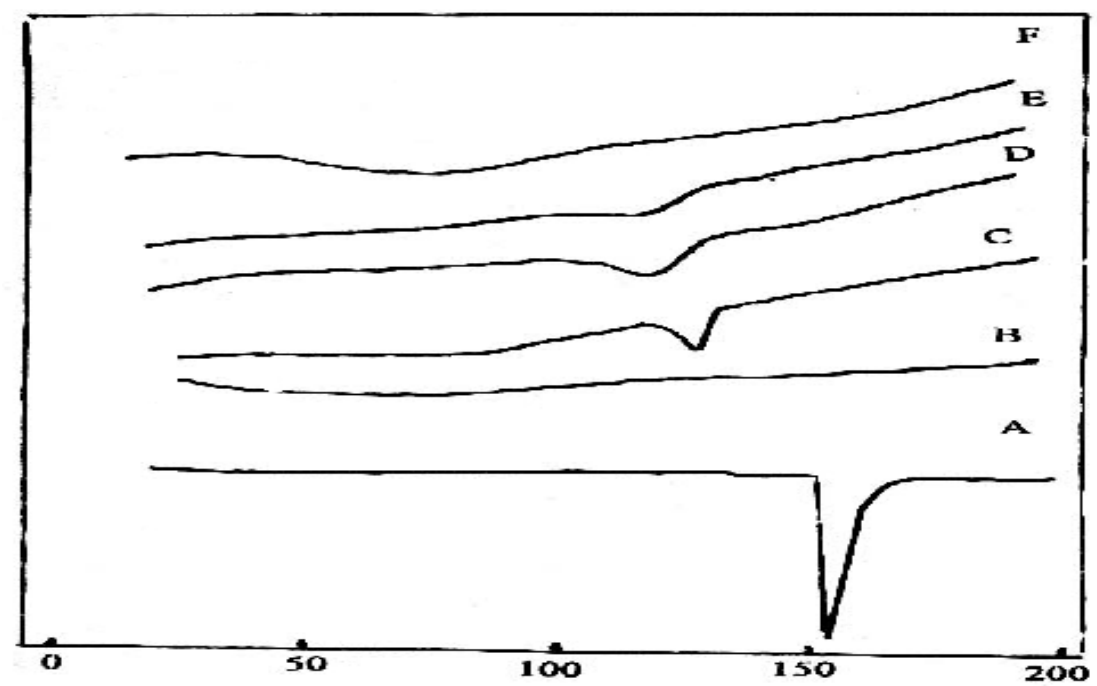

Fig. 3: DSC thermograms of naproxen/Florite R systems, where:

A- Plain drug B- Florite R

C- 1:1, drug/adsorbent physical mixture

D- 1:2, drug/adsorbent physical mixture

E- 1:1, drug/adsorbent loaded mixture

F- 1:2, drug/adsorbent loaded mixture

These results are in a good agreement with those obtained by Safwat et $a l^{11}$ who studied the effect of porous silica on the physicochemical properties of nystatine. Dissolution profiles of naproxen alone, its physical mixtures and adsorbates in different ratios with Florite R are illustrated in Figs. 4 and 5. The physical mixtures showed slightly faster drug release rate than plain drug. In addition, physical mixture and the adsorbate containing 1:2 ratio exhibited the highest dissolution rate compared to other ratios (Fig. 5). The mixing ratio of naproxen to Florite $\mathrm{R}$ in either physical or loaded mixtures has no significant effect on the dissolution behavior of the drug. These results indicated that the dissolution of naproxen from these systems depends on its crystalline state.

Regarding the adsorbate, when placed in the dissolution medium, drug molecules exhibited an amorphous state and can be released immediately into the dissolution medium. This finding was in agreement with that of Carli et al. ${ }^{17}$ 


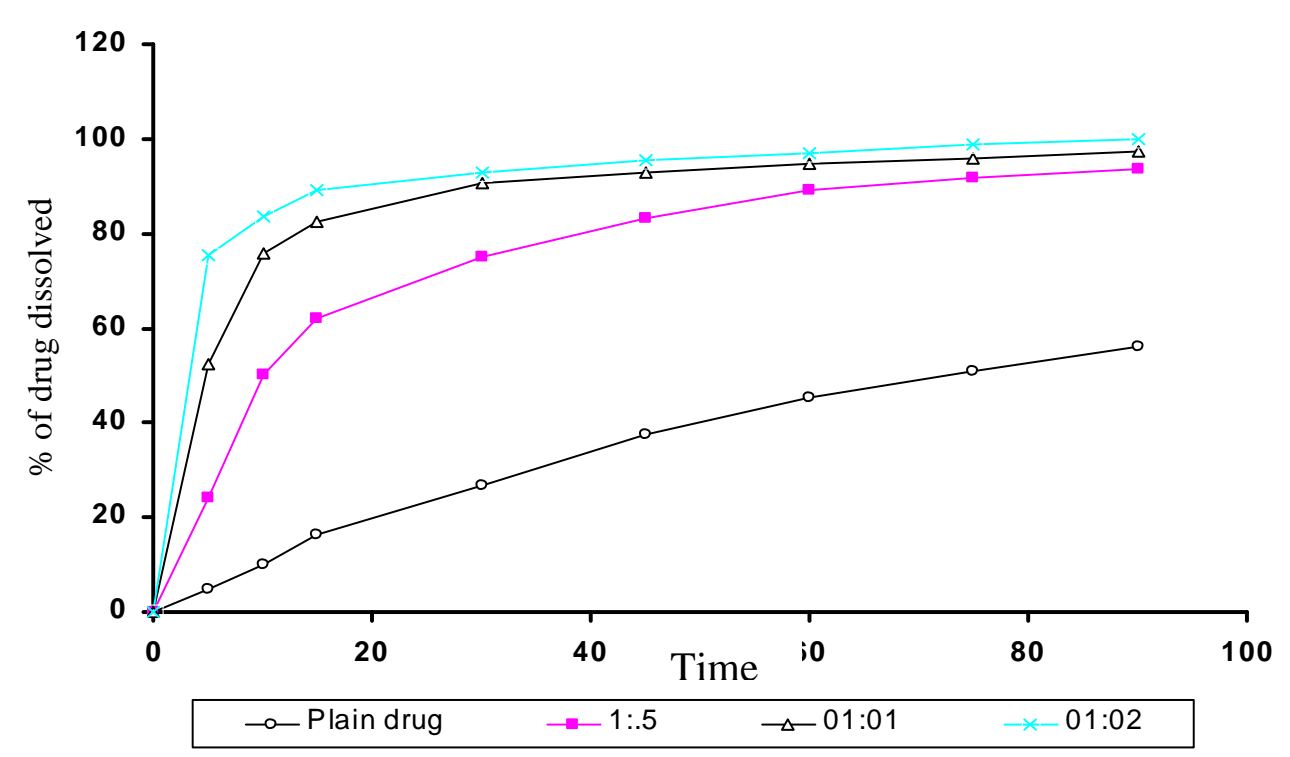

Fig. 4: Dissolution profiles of naproxen from physical mixtures with Florite $\mathrm{R}$ containing different drug: Florite R (w/w )ratios.

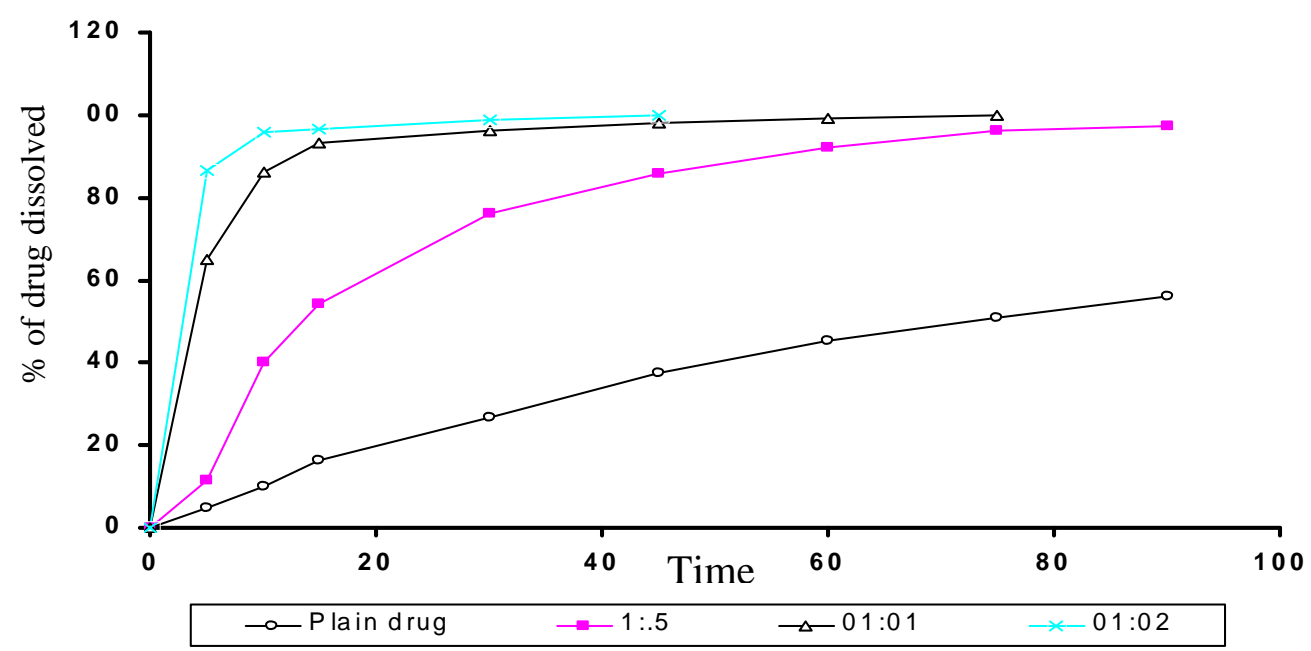

Fig. 5: Dissolution profiles of naproxen from adsorbates with Frolite $\mathrm{R}$ containing different drug: Florite R (w/w) ratios. 
The in-vitro release of naproxen from emulgels containing different gelling agents are illustrated in Figs. (6-9).

From the obtained results, it is so clear that sodium alginate gave the highest amount of released drug compared to other investigated gelling agents. The highest amount of released drug was obtained from sodium alginate and this may be due to the presence of sodium ion $\left(\mathrm{Na}^{+}\right)$, which converted naproxen into its sodium salt and subsequently gave higher drug solubility in the receiving medium $(\mathrm{pH}=5.03)$. This result is in a good agreement with that obtained by Atia. ${ }^{18}$ The difference in drug release between Sodium-alginate and Sodium-CMC emulgels may be attributed to the higher viscosity of Sodium-CMC.
The release data of naproxen from MC and HPMC emulgel bases are graphically illustrated in Figures 8 and 9 respectively, it is obvious that, the release of drug from HPMC is higher than that from MC and both more less than that obtained in case of sodium-alginate. The decrease in the amount of drug released from MC and HPMC may be attributed to the fact that, those bases are non-ionic ${ }^{19}$ and the drug will be in the free acid form which is less soluble in the receiving medium; $\mathrm{pH}=5.03$.

From the previous results it can be concluded that the extent of naproxen released from different investigated emulgel bases can be arranged in a descending order as follows:

Sodium-alginate $>$ HPMC $>$ sodium $\mathrm{CMC}>\mathrm{MC}$

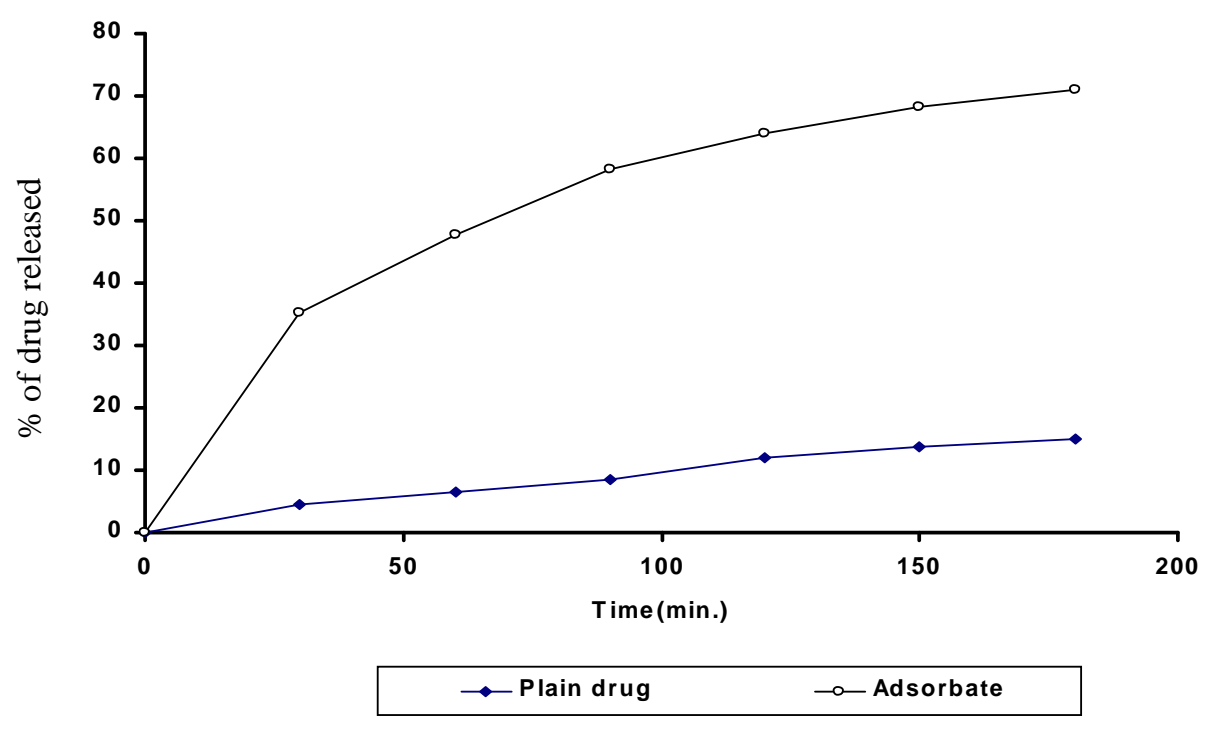

Fig. 6: Release profiles of naproxen from sodium alginate emulgel base (5\%). 


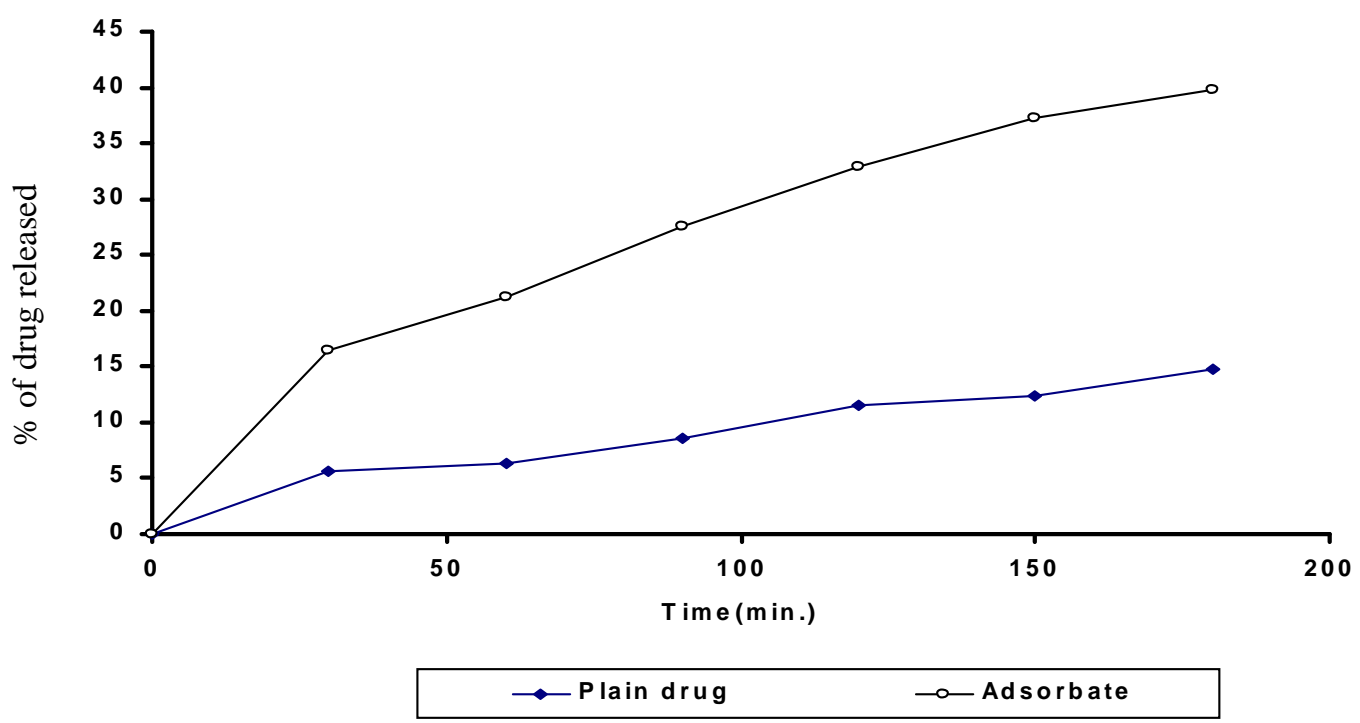

Fig. 7: Release profiles of naproxen from sodium CMC emulgel base (2\%).

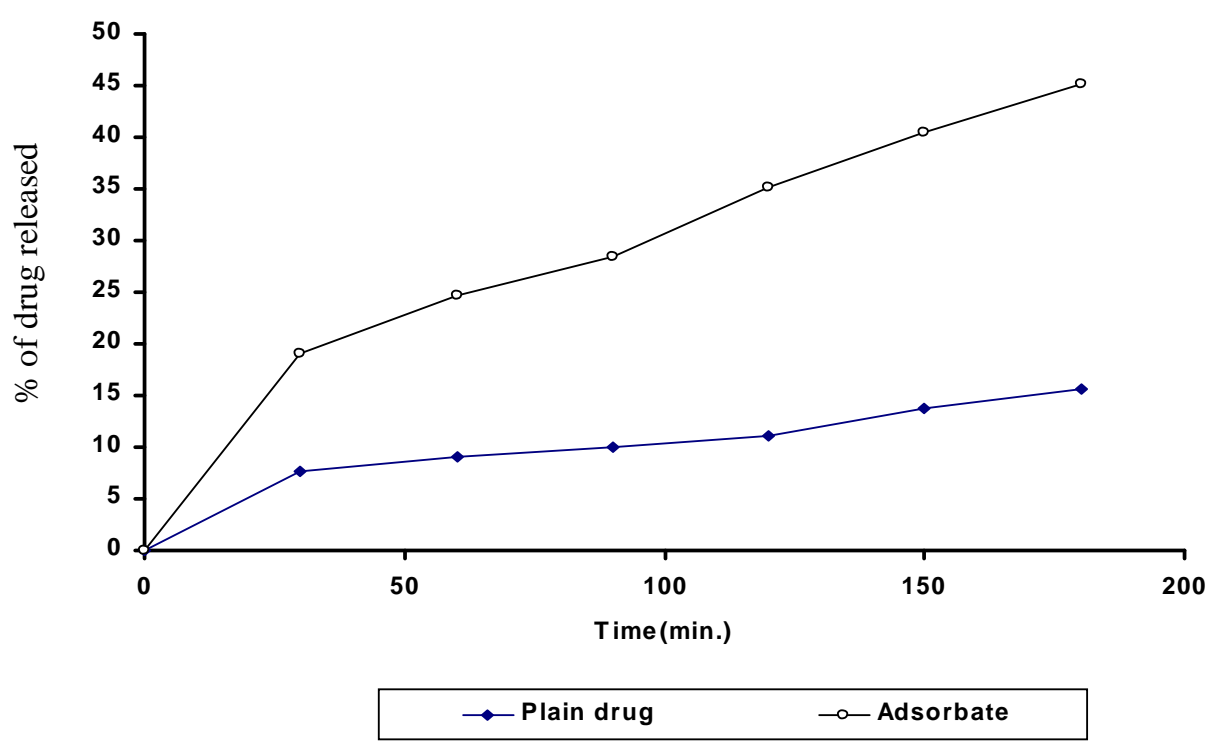

Fig. 8: Release profiles of naproxen from MC emulgel base (5\%). 


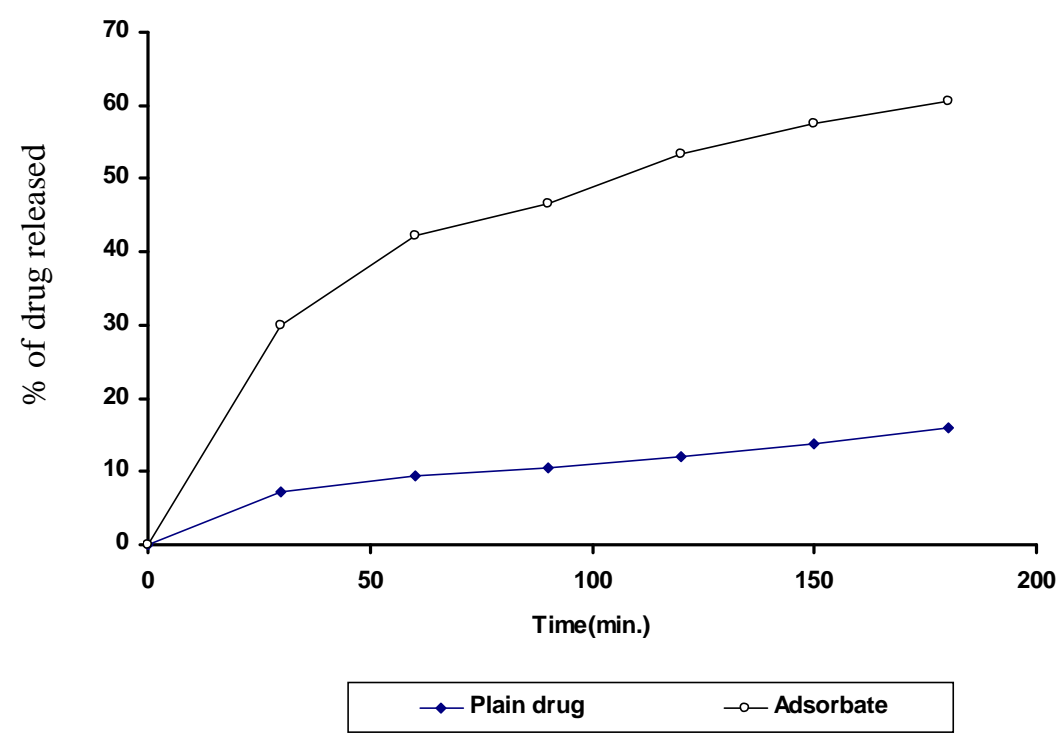

Fig. 9: Release profiles of naproxen from HPMC emulgel base (2.5\%).

\section{Conclusion}

From the obtained results it could be concluded that the release rate of naproxen from emulgel containing loaded mixtures was higher than that prepared using the plain drug. Also, the drug exhibited the highest release rate from the emulgel prepared by Na-alginate as compared to other gelling agents.

\section{REFERENCES}

1- Martinadale "The Extrapharmacopoeia" $33^{\text {th }}$ Ed. The Pharmaceutical Press, London, 2002, pp. 60-61.

2- "U. S. Pharmacopoeia" The national Formulary, $25^{\text {th }}$ Ed. NF 20, 2002, pp. 1191-1194.
3- T. Sato, A. Okada, K. Sekiguchi and Y. Tsuda, Chem. Pharm. Bull., 29, 2675 (1981).

4- H. Krasowska, II Farmaco, 31, 463 (1976)

5- H. Krasowska, Int. J. Pharm., 7 , 137 (1980).

6- T. Konno, Chem. Pharm. Bull., 38, 2003 (1990).

7- S. M. Alsaidan, A. A. Alsughayer, and A. G. Eshra, Drug Dev. Ind. Pharm., 24, 389 (1998).

8- A. Shaker, Bull. Pharm. Sci., Assiut University, 20, 1 (1997).

9- Y. Shu-Yang, C. Chun-Ren, L. Ming-Tao and C. Li-Chen, Drug Dev. Ind. Pharm., 23, 313 (1997).

10- R. K. Her, "The Chemistry of Silica", John Wiley, NY, USA, 1979, pp. 253-256 . 
11- S. M. Safwat, A. S. Ali, M. O. Ahmed and I. Abdel-Sabour, Bull. Pharm. Sci., Assiut University, 17, 139 (1994).

12- H.Y. Abdallah, N. Khalafalla, S.A. Khalil, Drug Dev. Ind. Pharm., 9, 795 (1983).

13- A. M. El-sayed, A. A. Shaker and A. A. Assi, STP Pharma Sci., 3, 319 (1993).

14- D. I. Friedman, J. S. Schwarz and M. Weisspapir, J. Pharm. Sci., 3, 84 (1995).

15- R. Nair, S. Gonen and S. W. Hoag, Int. J. Pharm., 240, 11 (2002).
16- M. Yoshioka, B. C. Hancock and G. Zografi, J. Pharm. Sci., 84, 983 (1995).

17- F. Carli, I. Colmbo, L. Magarotto and A. M.otto, $4^{\text {th }}$ Pharm. Technol. Conf., Edimb., V.K., (1984).

18- G. F. Atia, Master Thesis, Faculty of Pharmacy Zagazig University (1999).

19- C. M. Klech, "Gel and Gellies" in "Encyclopedia of Pharmaceutical Technology" J. Swarbrick and J. C. Boylan, Eds., Marcel Dekker, Inc., USA, Vol. 6 (1988). 